\title{
A local large deviation principle for inhomogeneous birth-death processes
}

\author{
N.D. Vvedenskaya ${ }^{1}$, A.V. Logachov ${ }^{2,3,4}$, Y.M. Suhov ${ }^{1,5}$, A.A. Yambartsev ${ }^{6}$
}

26 июня 2018 г.

\footnotetext{
${ }^{1}$ Dobrushin Laboratory, Institute for Information Transmission Problems, RAS, 19 Bol'shoi Karetnyi per, Moscow 127051, RF

E-mail: ndv@iitp.ru1

${ }^{2}$ Laboratory of Applied Mathematics, Novosibirsk State University, 2 Pirogov ul, Novosibirsk 630090, RF

${ }^{3}$ Laboratory of Probability Theory and Mathematical Statistics, Sobolev Institute of Mathematrics, Siberiian Branch of the RAS, 4 Koptyug ul, Novosibirsk 630090, RF

4 Statistics Division, Novosibirsk State University of Economics and Management, 56 Kamenskaya ul, Novosibirsk 630099, RF

E-mail: omboldovskaya@mail.ru2

${ }^{5}$ Math Dept, Penn State University, University Park, State College, PA 16802, USA.

E-mail: yms@statslab.cam.ac.uk

${ }^{6}$ Department of Statistics, Institute of Mathematics and Statistics, University of São Paulo, 1010 Rua do Matão, CEP 05508-090, São Paulo SP, Brazil

E-mail: yambar@gmail.com
}

\begin{abstract}
Аннотация
The paper considers a continuous-time birth-death process where the jump rate has an asymptotically polynomial dependence on the process position. We obtain a rough exponential asymptotics for the probability of excursions of a re-scaled process contained within a neighborhood of a given continuous non-negative function.

\footnotetext{
${ }^{1}$ The work by N.D. Vvedenskaya had been conducted at the IITP RAS under the support of the RSF (Project No14-50-00 150).

${ }^{2}$ The work by A.V. Logachov was supported by the RSF Grant (Project No18-11-00129).
} 


\section{Introduction}

In the modern literature on the large deviation principle, one considers various conditions for random processes guaranteeing a rough exponential asymptotics for probabilities of rare events. See, for example, [1 - 6]. In this paper we deal with birth-and-death Markov processes that are inhomogeneous in the state space: the rates of jumps are polynomially dependent on the position of the process. For these processes we obtain exponential asymptotics for the probabilities the normalized process to be in a neighborhood of a continuous function. Moreover, we provide this asymptotics both for ergodic processes and for transient (even exploding) processes.

The study of birth-and-death processes is of a certain mathematical interest and, moreover, is important for a number of applications. As examples, we can cite the information theory (encoding and storage of information, see [7]), biology and chemistry (models of growth and extinction in systems with multiple components, see [8], 9]), and economics (models of competitive production and pricing, [10], [11]).

Consider a continuous-time Markov process $\xi(t), t \geq 0$, with state space $\mathbb{Z}^{+} \cup\{\infty\}$, where $\mathbb{Z}^{+}=\{0\} \cup \mathbb{N}$. Let us assume that the process starts at 0 .

The evolution of the process $\xi(\cdot)$ is described as follows. For a given $t \geq 0$, let $\xi(t)=$ $x \in \mathbb{Z}^{+}$. The state of the random process does not change during the random time $\tau_{x}$ with exponential distribution with parameter $h(x)>0$. At the moment $t+\tau_{x}$ the process jumps to the states $x \pm 1$ with probabilities

$$
\mathbf{P}\left(\xi\left(t+\tau_{x}\right)=x+1\right)=\frac{\lambda(x)}{h(x)}, \quad \mathbf{P}\left(\xi\left(t+\tau_{x}\right)=x-1\right)=\frac{\mu(x)}{h(x)},
$$

correspondingly, where $\lambda(x)+\mu(x)=h(x), \lambda(x)>0$ when $x \in \mathbb{Z}^{+}$, and $\mu(x)>0$ for $x \in \mathbb{N}$.

Suppose that for $x=0$ the rates $\mu(x)=0, \lambda(x)=\lambda_{0}>0$ (i.e. the process cannot take negative values), and the following asymptotics hold true

$$
\lim _{x \rightarrow \infty} \frac{\lambda(x)}{P_{l} x^{l}}=\lim _{x \rightarrow \infty} \frac{\mu(x)}{Q_{m} x^{m}}=1
$$

where $P_{l}$ and $Q_{m}$ are positive constants, and $l \geq 0, m \geq 0, l \vee m>0$.

When $l \leq 1$, the existence of a Markov process with the above properties is established in the standard way, see for example [12], Ch. 17, §4, 5, and also [13], Ch. 2, § 5, Theorem 2.5.5, [14], Ch. 6, 7. When $l>1$ the process $\xi(\cdot)$, generally speaking, can go to infinity ("explode") during a random time, finite with probability one. There are two approaches to construct such processes. (1) One can stop the process at the random time point (the time of explosion); viz., see [15], Ch. 15, §4, [16], vol. 1, Ch 6, P. 365; vol. 2, P. 274. (2) One can 
extend the phase space $\mathbb{Z}^{+}$by adding an absorbing state (denoted by $\infty$ ); see, e.g., [17], Ch. $4, \S 48,[12$, Ch. 17, $\S 10$. In this paper we use the second approach.

The above class of random processes has been given the name birth-and-death processes; see, for example, [12], [17].

There exist conditions on $l$ and $m$ which are sufficient for explosion and non-explosion. For example, when $l>1$ and $m<l$, the process $\xi(\cdot)$ explodes, while if $m>l$ it does not. As references, cf. original papers [18], [19] and references within. See also [20], Ch. 23, § 7, [13], Ch. 2, $\S 5$, and [21], Ch.5, $\S 3$ (the last reference includes results for general Markov chains, not only for birth-and-death processes).

We are interested in the local large deviation principle (LLDP) for the family of scaled processes

$$
\xi_{T}(t)=\frac{\xi(t T)}{T}, 0 \leq t \leq 1,
$$

where $T>0$ is a parameter (see, [22], [23]). In a sense, the formulation and analysis of the LLDP should precede the study of other forms of the large deviation principle.

The validity of our results does not depend on whether or not the process $\xi(\cdot)$ explodes within a finite time. We focus on the asymptotics of the probability of the event that the trajectories of process $\xi_{T}(\cdot)$ to stay in a neighborhood of a continuous positive function given on the interval $[0,1]$. It means that we are working on the set of trajectories which do not tend to infinity in the time interval $[0, T]$. The considered probabilities are positive even if the process $\xi(\cdot)$ explodes (see equation ([6]) below).

Let $\mathbb{D}[0,1]$ denote the space of right-continuous functions with left-limit at eact $t \in[0,1]$. For any $f, g \in \mathbb{D}[0,1]$, set

$$
\rho(f, g)=\sup _{t \in[0,1]}|f(t)-g(t)|
$$

Definition 1.1 The family of random processes $\xi_{T}(\cdot)$ satisfies the LLDP on the set $G \subseteq$ $\mathbb{D}[0,1]$ with a rate functional $I=I(f): \mathbb{D}[0,1] \rightarrow[0, \infty)$ and a normalising function $T \in(0, \infty) \mapsto \psi(T)>0$ with $\lim _{T \rightarrow \infty} \psi(T)=\infty$ if, for any function $f \in G$, the following equality holds true:

$$
\begin{aligned}
& \lim _{\varepsilon \rightarrow 0} \limsup _{T \rightarrow \infty} \frac{1}{\psi(T)} \ln \mathbf{P}\left(\xi_{T}(\cdot) \in U_{\varepsilon}(f)\right) \\
& \quad=\lim _{\varepsilon \rightarrow 0} \liminf _{T \rightarrow \infty} \frac{1}{\psi(T)} \ln \mathbf{P}\left(\xi_{T}(\cdot) \in U_{\varepsilon}(f)\right)=-I(f) .
\end{aligned}
$$

Here

$$
U_{\varepsilon}(f)=\{g \in \mathbb{D}[0,1]: \rho(f, g)<\varepsilon\} .
$$


In the framework of Definition 1.1 there are various cases to consider. We separate three cases: 1) $l>m, 2) l<m$ and $l=m$.

Note that the case $m=1, l=0$, follows from [10] (where a two dimensional Markov process is treated). A similar result is obtained in [24] for solutions of stochastic differential Ito's equations. The classical case $l=m=0, \varphi(T)=T$ follows, for example, from [25].

In this paper we use the approach developed in [10]. We would like to note that the large deviation principle for the sequence of processes $\xi_{T}(\cdot)$ in space $\mathbb{D}[0,1]$ with Skorohod metric cannot be obtained even for non-exploding processes: one can show that the corresponding family of measures is not exponentially dense, except for the case $l=m=0$.

The paper is organized as follows: in $\S 2$ we introduce our definitions and the system of notation, as well as the main result (Theorem 2.1) and key lemmas. In $\S 3$ we prove Theorem 2.1 and key lemmas. In $\S 4$ (the Appendix) some auxiliary technical assertions are established.

\section{Main results, definitions}

Let $F$ denote the set of functions $f(t) \in \mathbb{C}[0,1]$ such that $f(0)=0$ and $f(t)>0$ as $0<t \leq 1$.

Theorem 2.1 Let the conditions (1) and (2) be fulfilled. Than the random processes sequence $\xi_{T}(\cdot)$ on $F$ fulfills the following LLDP:

a) If $l>m$ then the normalizing function $\psi(T)=T^{l+1}$, and the rate functional has the form

$$
I(f)=P_{l} \int_{0}^{1} f^{l}(t) d t, \quad f \in F .
$$

b) If $l=m$ and $P_{l} \neq Q_{m}$ then $\psi(T)=T^{l+1}$ and

$$
I(f)=\left(\sqrt{P_{l}}-\sqrt{Q_{m}}\right)^{2} \int_{0}^{1} f^{l}(t) d t, \quad f \in F .
$$

c) If $l<m$ then $\psi(T)=T^{m+1}$ and

$$
I(f)=Q_{m} \int_{0}^{1} f^{m}(t) d t, \quad f \in F .
$$

The case where $l=m$ and $P_{l}=Q_{m}$ needs a different normalization; we do not discuss it in this paper.

Consider a space- and time-homogenous Markov process $\zeta(t), t \in[0, T]$, on the phase space $\mathbb{Z}$, where the jump rate is equal to 1 , and the jump size is \pm 1 , occurring with probability $1 / 2$. 
Denote by $X_{T}$ the set of all right-continuous step-functions with a finite number of \pm 1 -jumps on $[0, T]$.

Lemma 2.2 For any given $T$, the distribution $\mathbf{P}_{T}^{(\xi)}\left(\cdot \cap X_{T}\right)$ of process $\xi(\cdot)$ on $X_{T}$ is absolutely continuous with respect to the distribution $\mathbf{P}_{T}^{(\zeta)}$ of process $\zeta(\cdot)$ on $X_{T}$. The corresponding density (the Radon-Nikodym derivative) $\mathfrak{p}_{T}(u)=\frac{d \mathbf{P}_{T}^{(\xi)}}{d \mathbf{P}_{T}^{(\zeta)}}(u), u \in X_{T}$, has the form

$$
\mathfrak{p}_{T}(u)=\left\{\begin{array}{cl}
2^{N_{T}(u)}\left(\prod_{i=1}^{N_{T}(u)} e^{-\left(h\left(u\left(t_{i-1}\right)\right)-1\right) \tau_{i}} \nu\left(u\left(t_{i-1}\right), u\left(t_{i}\right)\right)\right) \\
\times e^{-\left(h\left(u\left(t_{N_{T}(u)}\right)-1\right)\right)\left(T-t_{N_{T}(u)}\right)}, & \text { if } N_{T}(u) \geq 1, \\
e^{-(h(0)-1) T}, & \text { if } N_{T}(u)=0 .
\end{array}\right.
$$

Here it is supposed that the function $u(\cdot)$ on $[0, T]$ has exactly $N_{T}(u)$ jumps at the time points $t_{1}, t_{2}, \ldots, t_{N_{T}(u)}$ where $0=t_{0}<t_{1}<\ldots<t_{N_{T}(u)} \leq T, \tau_{i}=t_{i}-t_{i-1}$. Moreover

$$
\nu\left(u\left(t_{i-1}\right), u\left(t_{i}\right)\right)= \begin{cases}\lambda\left(u\left(t_{i-1}\right)\right), & \text { if } u\left(t_{i}\right)-u\left(t_{i-1}\right)=1 \\ \mu\left(u\left(t_{i-1}\right)\right), & \text { if } u\left(t_{i}\right)-u\left(t_{i-1}\right)=-1\end{cases}
$$

Observe that the probability $\mathbf{P}\left(\xi(\cdot) \in X_{T}\right)$ in Lemma 2.2 is allowed to be less then 1. (Clearly, this probability is positive.) The same density $\mathfrak{p}_{T}$ was used in [10].

Let us denote by $N_{T}(\zeta)$ the random number of jumps in process $\zeta(\cdot)$ on interval $[0, T]$. The assertion of Lemma 2.2 is equivalent to the fact that for any measurable set $G \subseteq X_{T}$

$$
\mathbf{P}(\xi(\cdot) \in G)=e^{T} \mathbf{E}\left(e^{-A_{T}(\zeta)} e^{B_{T}(\zeta)+N_{T}(\zeta) \ln 2} ; \zeta(\cdot) \in G\right)
$$

We set

$$
\begin{aligned}
& A_{T}(\zeta)=\int_{0}^{T} h(\zeta(t)) d t \\
& = \begin{cases}\sum_{i=1}^{N_{T}(\zeta)} h\left(\zeta\left(t_{i-1}\right)\right) \tau_{i}+h\left(\zeta\left(t_{N_{T}(\zeta)}\right)\right)\left(T-t_{N_{T}(\zeta)}\right), & \text { if } N_{T}(\zeta) \geq 1, \\
h(0) T, & \text { if } N_{T}(\zeta)=0 ;\end{cases} \\
& B_{T}(\zeta)= \begin{cases}\sum_{i=1}^{N_{T}(\zeta)} \ln \left(\nu\left(\zeta\left(t_{i-1}\right), \zeta\left(t_{i}\right)\right)\right), & \text { if } N_{T}(\zeta) \geq 1, \\
0, & \text { if } N_{T}(\zeta)=0 .\end{cases}
\end{aligned}
$$

The expressions in (7) specify, in our context, the statement of the Radon-Nikodym theorem (see, e.g., [26], Theorem 2, sec. III, ch. 10). The expressions (7) are used for analysing the asymptotical behaviour of the logarithm of probability $\mathbf{P}\left(\xi_{T}(\cdot) \in U_{\varepsilon}(f)\right), f \in F$. 
Theorem 2.1 indicates that for $l \neq m$ the main contribution into the asymptotics is brought by $A_{T}(\zeta)$, whereas in the case $l=m$ the asymptotics involves both $A_{T}(\zeta)$ and $B_{T}(\zeta)$.

Consider the family of scaled processes

$$
\zeta_{T}(t)=\frac{\zeta(t T)}{T}, \quad t \in[0,1]
$$

Let $k_{+}$and $k_{-}$denote the number of positive and negative jumps in $\zeta_{T}(\cdot)$ and set $L=k_{+}-k_{-}$.

For $\zeta_{T}(\cdot) \in U_{\varepsilon}(f)$ we have the inequality

$$
f(1)-\varepsilon \leq \zeta_{T}(1) \leq f(1)+\varepsilon
$$

The jumps in $\zeta_{T}(\cdot)$ are $\pm 1 / T$, therefore (9) yields the inequalities

$$
(f(1)-\varepsilon) T \leq L \leq(f(1)+\varepsilon) T
$$

With these definitions and observations we can write:

$$
k_{+}+k_{-}=N_{T}(\zeta), \quad k_{+}=\frac{N_{T}(\zeta)+L}{2}, \quad k_{-}=\frac{N_{T}(\zeta)-L}{2} .
$$

For brevity, we write below $\xi_{T}, \zeta_{T}$ and $A_{T}, B_{T}$ instead of $\xi_{T}(\cdot), \zeta_{T}(\cdot)$ and $A_{T}(\zeta), B_{T}(\zeta)$. Also set: $v=\max (l, m)$.

Lemma 2.3 Let $f \in F$. In case $l \neq m$ we have

$$
\limsup _{\varepsilon \rightarrow 0} \limsup _{T \rightarrow \infty} \frac{1}{T^{v+1}} \ln \mathbf{E}\left(e^{B_{T}+N_{T}(\zeta) \ln 2} ; \zeta_{T} \in U_{\varepsilon}(f)\right) \leq 0
$$

whereas in case $l=m$

$$
\lim _{\varepsilon \rightarrow 0} \limsup _{T \rightarrow \infty} \frac{1}{T^{l+1}} \ln \mathbf{E}\left(e^{B_{T}+N_{T}(\zeta) \ln 2} ; \zeta_{T} \in U_{\varepsilon}(f)\right) \leq 2 \sqrt{P_{l} Q_{m}} \int_{0}^{1} f^{l}(s) d s,
$$

Lemma 2.4 For $f \in F$, in case $l \neq m$

$$
\lim _{\varepsilon \rightarrow 0} \liminf _{T \rightarrow \infty} \frac{1}{T^{v+1}} \ln \mathbf{E}\left(e^{B_{T}+N_{T}(\zeta) \ln 2} ; \zeta_{T} \in U_{\varepsilon}(f)\right) \geq 0,
$$

and in case $l=m$

$$
\lim _{\varepsilon \rightarrow 0} \liminf _{T \rightarrow \infty} \frac{1}{T^{l+1}} \ln \mathbf{E}\left(e^{B_{T}+N_{T}(\zeta) \ln 2} ; \zeta_{T} \in U_{\varepsilon}(f)\right) \geq 2 \sqrt{P_{l} Q_{m}} \int_{0}^{1} f^{l}(s) d s .
$$




\section{Proofs of Theorem 2.1 and Lemmas $2.2-2.4$}

In what follows, $\boldsymbol{\Delta}$ marks the end of a proof.

Proof of Theorem 2.1 We are going to get the LLDP for functions $f \in F$. First let us estimate the quantity $A_{T}$. Fix a value $\varepsilon>0$ until a further notice.

From equation (8) it follows that

$$
A_{T}:=\int_{0}^{T} h(\zeta(t)) d t=T \int_{0}^{1} h\left(T \zeta_{T}(s)\right) d s .
$$

If $\zeta_{T} \in U_{\varepsilon}(f)$ then

$$
(f(s)-\varepsilon) \leq \zeta_{T}(s) \leq(f(s)+\varepsilon) .
$$

Let $\delta \in(0,1)$ be also fixed for the time being and denote $m_{\delta}:=\min _{t \in[\delta, 1]} f(t)$. Here $m_{\delta}>0$ for $f \in F$. Therefore, $k_{0}=m_{\delta}-\varepsilon>0$ when $\varepsilon$ is sufficiently small.

Let us estimate $A_{T}$ on the set of trajectories where inequality (12) is valid. From (12) it follows that $T \zeta_{T}(s) \geq k_{0} T$ for $s \in[\delta, 1]$. Therefore, by virtue of condition (2), for any $\gamma_{0} \in(0,1)$ and $s \in[\delta, 1]$, for $T$ large enough we have the inequalities

$$
\begin{gathered}
1-\gamma_{0} \leq \frac{h\left(T \zeta_{T}(s)\right)}{P_{l}\left(T \zeta_{T}(s)\right)^{l}} \leq 1+\gamma_{0} \quad \text { in case } l>m, \\
1-\gamma_{0} \leq \frac{h\left(T \zeta_{T}(s)\right)}{\left(P_{l}+Q_{m}\right)\left(T \zeta_{T}(s)\right)^{l}} \leq 1+\gamma_{0} \text { in case } l=m,
\end{gathered}
$$

and

$$
1-\gamma_{0} \leq \frac{h\left(T \zeta_{T}(s)\right)}{Q_{m}\left(T \zeta_{T}(s)\right)^{m}} \leq 1+\gamma_{0} \quad \text { in case } \quad l<m
$$

Consider the case $l>m$. Owing to (12) and (13), for $T$ sufficiently large, we get

$$
\begin{aligned}
& T \int_{\delta}^{1}\left(1-\gamma_{0}\right) P_{l}(T(f(s)-\varepsilon))^{l} d s \leq A_{T} \\
& \quad \leq T \int_{0}^{\delta} h\left(T \zeta_{T}(s)\right) d s+T \int_{\delta}^{1}\left(1+\gamma_{0}\right) P_{l}(T(f(s)+\varepsilon))^{l} d s
\end{aligned}
$$

Set $M:=\max \left(\max _{t \in[0,1]} f(t), 1\right)$. By using (12), for $T$ large enough we have that

$$
h\left(T \zeta_{T}(s)\right) \leq\left(1+\gamma_{0}\right) P_{l}(T(M+\varepsilon))^{l} .
$$

Consequently, from (16) we obtain the inequality

$$
\begin{aligned}
& T^{l+1} P_{l} \int_{\delta}^{1}\left(1-\gamma_{0}\right)(f(s)-\varepsilon)^{l} d s \leq A_{T} \\
& \quad \leq T^{l+1} P_{l} \delta\left(1+\gamma_{0}\right)(M+\varepsilon)^{l}+T^{l+1} P_{l} \int_{\delta}^{1}\left(1+\gamma_{0}\right)(f(s)+\varepsilon)^{l} d s .
\end{aligned}
$$


By using the bound (17) and equation (77), we get the following:

$$
\begin{aligned}
\exp [- & \left.T^{l+1} P_{l} \int_{\delta}^{1}\left(1-\gamma_{0}\right)(f(s)-\varepsilon)^{l} d s\right] e^{T} \mathbf{E}\left(e^{B_{T}+N_{T}(\zeta) \ln 2} ; \zeta_{T} \in U_{\varepsilon}(f)\right) \\
\geq & \mathbf{P}\left(\xi_{T}(\cdot) \in U_{\varepsilon}(f)\right)=e^{T} \mathbf{E}\left(e^{-A_{T}} e^{B_{T}+N_{T}(\zeta) \ln 2} ; \zeta_{T} \in U_{\varepsilon}(f)\right) \\
\geq & \exp \left[-T^{l+1} P_{l} \delta\left(1+\gamma_{0}\right)(M+\varepsilon)^{l}-T^{l+1} P_{l} \int_{\delta}^{1}\left(1+\gamma_{0}\right)(f(s)+\varepsilon)^{l} d s\right] \\
& \times e^{T} \mathbf{E}\left(e^{B_{T}+N_{T}(\zeta) \ln 2} ; \zeta_{T} \in U_{\varepsilon}(f)\right) .
\end{aligned}
$$

Further, by virtue of (18)

$$
\begin{aligned}
& -P_{l} \int_{\delta}^{1}\left(1-\gamma_{0}\right)(f(s)-\varepsilon)^{l} d s+\limsup _{T \rightarrow \infty} \frac{1}{T^{l+1}} \ln \mathbf{E}\left(e^{B_{T}+N_{T}(\zeta) \ln 2} ; \zeta_{T} \in U_{\varepsilon}(f)\right) \\
& \geq \limsup _{T \rightarrow \infty} \frac{1}{T^{l+1}} \ln \mathbf{P}\left(\xi_{T} \in U_{\varepsilon}(f)\right) \geq \liminf _{T \rightarrow \infty} \frac{1}{T^{l+1}} \ln \mathbf{P}\left(\xi_{T} \in U_{\varepsilon}(f)\right) \\
& \geq-P_{l} \delta\left(1+\gamma_{0}\right)(M+\varepsilon)^{l}-P_{l} \int_{\delta}^{1}\left(1+\gamma_{0}\right)(f(s)+\varepsilon)^{l} d s \\
& \quad+\liminf _{T \rightarrow \infty} \frac{1}{T^{l+1}} \ln \mathbf{E}\left(e^{B_{T}+N_{T}(\zeta) \ln 2} ; \zeta_{T} \in U_{\varepsilon}(f)\right) .
\end{aligned}
$$

Next, from (19) it follows that

$$
\begin{aligned}
- & P_{l} \int_{\delta}^{1}\left(1-\gamma_{0}\right) f^{l}(s) d s+\lim _{\varepsilon \rightarrow 0} \limsup _{T \rightarrow \infty} \frac{1}{T^{l+1}} \ln \mathbf{E}\left(e^{B_{T}+N_{T}(\zeta) \ln 2} ; \zeta_{T} \in U_{\varepsilon}(f)\right) \\
\geq \lim _{\varepsilon \rightarrow 0} \limsup _{T \rightarrow \infty} \frac{1}{T^{l+1}} \ln \mathbf{P}\left(\xi_{T}(\cdot)\right. & \left.\in U_{\varepsilon}(f)\right) \geq \lim _{\varepsilon \rightarrow 0} \liminf _{T \rightarrow \infty} \frac{1}{T^{l+1}} \ln \mathbf{P}\left(\xi_{T}(\cdot) \in U_{\varepsilon}(f)\right) \\
\geq-P_{l} \delta\left(1+\gamma_{0}\right) M^{l}-P_{l} \int_{0}^{1}(1 & \left.+\gamma_{0}\right) f^{l}(s) d s \\
& +\lim _{\varepsilon \rightarrow 0} \liminf _{T \rightarrow \infty} \frac{1}{T^{l+1}} \ln \mathbf{E}\left(e^{B_{T}+N_{T}(\zeta) \ln 2} ; \zeta_{T} \in U_{\varepsilon}(f)\right) .
\end{aligned}
$$

Note that the inequatity (20) is valid for all $\gamma_{0}, \delta>0$. Letting $\gamma_{0} \rightarrow 0$ and $\delta \rightarrow 0$ we get that

$$
\begin{aligned}
- & P_{l} \int_{0}^{1} f^{l}(s) d s+\lim _{\varepsilon \rightarrow 0} \limsup _{T \rightarrow \infty} \frac{1}{T^{l+1}} \ln \mathbf{E}\left(e^{B_{T}+N_{T}(\zeta) \ln 2} ; \zeta_{T} \in U_{\varepsilon}(f)\right) \\
& \geq \lim _{\varepsilon \rightarrow 0} \limsup _{T \rightarrow \infty} \frac{1}{T^{l+1}} \ln \mathbf{P}\left(\xi_{T}(\cdot) \in U_{\varepsilon}(f)\right) \geq \lim _{\varepsilon \rightarrow 0} \liminf _{T \rightarrow \infty} \frac{1}{T^{l+1}} \ln \mathbf{P}\left(\xi_{T}(\cdot) \in U_{\varepsilon}(f)\right) \\
& \geq-P_{l} \int_{0}^{1} f^{l}(s) d s+\lim _{\varepsilon \rightarrow 0} \liminf _{T \rightarrow \infty} \frac{1}{T^{l+1}} \ln \mathbf{E}\left(e^{B_{T}+N_{T}(\zeta) \ln 2} ; \zeta_{T} \in U_{\varepsilon}(f)\right) .
\end{aligned}
$$

In a similar way, by using (14) and (15) we obtain inequalities for the case $l=m$ :

$$
\begin{aligned}
& -\left(P_{l}+Q_{m}\right) \int_{0}^{1} f^{l}(s) d s+\lim _{\varepsilon \rightarrow 0} \limsup _{T \rightarrow \infty} \frac{1}{T^{l+1}} \ln \mathbf{E}\left(e^{B_{T}+N_{T}(\zeta) \ln 2} ; \zeta_{T} \in U_{\varepsilon}(f)\right) \\
& \geq \lim _{\varepsilon \rightarrow 0} \limsup _{T \rightarrow \infty} \frac{1}{T^{l+1}} \ln \mathbf{P}\left(\xi_{T} \in U_{\varepsilon}(f)\right) \geq \lim _{\varepsilon \rightarrow 0} \liminf _{T \rightarrow \infty} \frac{1}{T^{l+1}} \ln \mathbf{P}\left(\xi_{T} \in U_{\varepsilon}(f)\right) \\
& \geq-\left(P_{l}+Q_{m}\right) \int_{0}^{1} f^{l}(s) d s+\lim _{\varepsilon \rightarrow 0} \liminf _{T \rightarrow \infty} \frac{1}{T^{l+1}} \ln \mathbf{E}\left(e^{B_{T}+N_{T}(\zeta) \ln 2} ; \zeta_{T} \in U_{\varepsilon}(f)\right),
\end{aligned}
$$


and for the case $l<m$ :

$$
\begin{aligned}
& -Q_{m} \int_{0}^{1} f^{m}(s) d s+\lim _{\varepsilon \rightarrow 0} \limsup _{T \rightarrow \infty} \frac{1}{T^{m+1}} \ln \mathbf{E}\left(e^{B_{T}+N_{T}(\zeta) \ln 2} ; \zeta_{T} \in U_{\varepsilon}(f)\right) \\
& \geq \lim _{\varepsilon \rightarrow 0} \limsup _{T \rightarrow \infty} \frac{1}{T^{m+1}} \ln \mathbf{P}\left(\xi_{T}(\cdot) \in U_{\varepsilon}(f)\right) \geq \lim _{\varepsilon \rightarrow 0} \liminf _{T \rightarrow \infty} \frac{1}{T^{m+1}} \ln \mathbf{P}\left(\xi_{T}(\cdot) \in U_{\varepsilon}(f)\right) \\
& \geq-Q_{m} \int_{0}^{1} f^{m}(s) d s+\lim _{\varepsilon \rightarrow 0} \liminf _{T \rightarrow \infty} \frac{1}{T^{m+1}} \ln \mathbf{E}\left(e^{B_{T}+N_{T}(\zeta) \ln 2} ; \zeta_{T} \in U_{\varepsilon}(f)\right) .
\end{aligned}
$$

Observe that in the course if deducing the estimates (21), (22) and (23) the limit $T \rightarrow \infty$ precedes the limit $\varepsilon \rightarrow 0$.

Applying Lemmas 2.3 and 2.4 to (21) -(23) completes the proof of the LLDP for the functions $\in F$.

Remark 3.1 The above argument allows us to extend the assertion of Theorem 2.1 to the set of functions $f \in \mathrm{C}[0,1]$ with $f(0)=0, f(t) \geq 0$ for $0<t \leq 1$ and $f(t)=0$ at finitely many points in $[0,1]$.

Remark 3.2 For the Yule process (a process of pure birth with $l>0, P_{l}>0$ and $\mu(x) \equiv 0$, see e.g. [12]), the rate functional has the form

$$
I(f)=P_{l} \int_{0}^{1} f^{l}(t) d t, \quad f \in F_{M},
$$

where $F_{M}$ is a set of not-decreasing continuous functions $f(t)$ on $[0,1]$ with $f(0)=0$.

Proof of Lemma 2.2. Let $N_{T}(\xi)$ be the number of jumps in process $\xi$ in the time interval $[0, T]$. In the course of the proof we work on the event that the trajectoriy of $\xi$ belongs to $X_{T}$, i.e., that $N_{T}<\infty$. This event has a positive probability.

As was mentioned earlier, the statement of the lemma means that for any measurable set $G \subseteq X_{T}$ the equality (7) is valid. Denote by $X_{T}^{(n)}$ a set of functions $u \in X_{T}$ with $N_{T}(u)=n, n=0,1, \ldots$ Consider one-to-one mapping

$$
u \in X_{T}^{(n)} \mapsto\left(t_{1}, \ldots, t_{n} ; \Delta_{1}, \ldots, \Delta_{n}\right) \in \mathfrak{X}_{T}^{(n)}=[0, T]_{<}^{n} \times\{+1,-1\}^{n}, n=1,2 \ldots
$$

Here $t_{1}, \ldots, t_{n}$ is a sequence of jump times for function $u$ in $[0, T], \Delta_{i}$ is a size of jump $u\left(t_{i}\right)-$ $u\left(t_{i-1}\right)$ (with $\left.\Delta_{1}=u\left(t_{1}\right)\right)$. Next $[0, T]_{<}^{n}$ stands for an $n$-dimensional simplex $\left\{\left(t_{1}, \ldots, t_{n}\right)\right.$ : $\left.0<t_{1}<\ldots t_{n} \leq T\right\}$.

The probabilities $\mathbf{P}(\xi(\cdot) \in G)$ and $\mathbf{P}(\zeta(\cdot) \in G)$ are determiined by

a) the respective densities $\mathfrak{f}_{\xi}$ and $\mathfrak{f}_{\zeta}$ relative to the summation measure $\sum_{n \geq 1} \prod_{j=1}^{n} \mathrm{~d} t_{j}$ on $\mathfrak{X}_{T}:=\bigcup_{n \geq 1} \mathfrak{X}_{T}^{(n)}\left(\right.$ here $t_{0}=0$ as $\left.j=1\right)$, and 
b) the probabilities $\mathbf{P}(\xi(t)=0,0 \leq t \leq T)=e^{-\lambda(0) T}, \mathbf{P}(\zeta(t)=0,0 \leq t \leq T)=e^{-T}$.

The densities $\mathfrak{f}_{\xi}$ and $\mathfrak{f}_{\zeta}$ are of form

$$
\mathfrak{f}_{\xi}\left(t_{1}, \ldots, t_{n} ; \Delta_{1}, \ldots, \Delta_{n}\right)=\left(\prod_{i=1}^{n} \nu\left(x_{i-1}, x_{i}\right) e^{-h\left(x_{i-1}\right) \tau_{i}}\right) e^{-h\left(x_{n}\right)\left(T-t_{n}\right)},
$$

and

$$
\mathfrak{f}_{\zeta}\left(t_{1}, \ldots, t_{n} ; \Delta_{1}, \ldots, \Delta_{n}\right)=2^{-n}\left(\prod_{i=1}^{n} e^{-\tau_{i}}\right) e^{-\left(T-t_{n}\right)}
$$

where $x_{0}=0, x_{i}=\sum_{j=1}^{i} \Delta_{j}, i=1, \ldots, n$.

Each factor $\nu\left(x_{i-1}, x_{i}\right) e^{-h\left(x_{i-1}\right) \tau_{i}}$ in (25) gives the probability density $h\left(x_{i-1}\right) e^{-h\left(x_{i-1}\right) \tau_{i}}$ for the time the process $\xi$ spent at state $x_{i-1}$ multiplied by the probability $\nu\left(x_{i-1}, x_{i}\right) / h\left(x_{i-1}\right)$ of a jump from $x_{i-1}$ to $x_{i}$. The factor $e^{-h\left(x_{n}\right)\left(T-t_{n}\right)}$ is the probability to stay at $x_{n}$ until time T. A similar meaning is attributed to the factors $\frac{1}{2} e^{-\tau_{i}}$ and $e^{-\left(T-\text { ttostaya }_{n}\right)}$. The products of terms in (25) and (26) reflect the Markovian character of both processes.

The Radon-Nikodym derivative $\mathfrak{p}_{T}=\mathrm{d} \mathbf{P}_{T}^{(\xi)}\left(\cdot \cap X_{T}\right) / \mathrm{d} \mathbf{P}_{T}^{(\zeta)}$ in (6) is a ratio $\mathfrak{f}_{\xi} / \mathfrak{f}_{\zeta}$ because the mapping $X_{T}^{(n)} \rightarrow \mathfrak{X}_{T}^{(n)}$ is one-to-one. The Radon-Nikodym theorem can be applied here as both densities $\mathfrak{f}_{\xi}$ and $\mathfrak{f}_{\zeta}$ are positive on $\mathfrak{X}_{T}^{(n)} \sum_{n \geq 1} \prod_{j=1}^{n} \mathrm{~d} t_{j}$ and measure $\mathfrak{X}_{T}$ is finite (for formulation and proof of Radon-Nicodim Theorem see, e.g., [26], Chapter III, Section 10, Theorem 2, or [27], Theorem 6.10).

Proof of Lemma 2.3. First, we upper-bound the expected value $\mathbf{E}\left(e^{B_{T}+N_{T}(\zeta) \ln 2} ; \zeta_{T} \in\right.$ $\left.U_{\varepsilon}(f)\right)$.

Given $a>1$ we write:

$$
\begin{aligned}
& \mathbf{E}\left(e^{B_{T}+N_{T}(\zeta) \ln 2} ; \zeta_{T} \in U_{\varepsilon}(f)\right):=E_{1}+E_{2}, \\
& E_{1}:=\mathbf{E}\left(e^{B_{T}+N_{T}(\zeta) \ln 2} ; \zeta_{T} \in U_{\varepsilon}(f) ; N_{T}(\zeta) \leq T^{a}\right), \\
& E_{2}:=\mathbf{E}\left(e^{B_{T}+N_{T}(\zeta) \ln 2} ; \zeta_{T} \in U_{\varepsilon}(f) ; N_{T}(\zeta)>T^{a}\right) .
\end{aligned}
$$

Let us bound $E_{1}$ from above. If $\zeta_{T} \in U_{\varepsilon}(f)$ and $N_{T}(\zeta) \leq T^{a}$ then, by virtue of (2), it follows that for any $\gamma_{1}>0$ and $T$ large enough,

$$
\begin{aligned}
B_{T} & =\sum_{i=1}^{N_{T}(\zeta)} \ln \left(\nu\left(\zeta\left(t_{i-1}\right), \zeta\left(t_{i}\right)\right)\right. \\
& \leq T^{a}\left(\ln \left(P_{l} T^{l}(M+\varepsilon)^{l}\left(1+\gamma_{1}\right)\right)+\ln \left(Q_{m} T^{m}(M+\varepsilon)^{m}\left(1+\gamma_{1}\right)\right)\right) .
\end{aligned}
$$

Here, as before, $M=\max \left[\max _{t \in[0,1]} f(t), 1\right]$.

Set $k_{1}=P_{l} Q_{m}(M+\varepsilon)^{l+m}\left(1+\gamma_{1}\right)^{2}$. Then the following inequality is fulfilled:

$$
E_{1} \leq \exp \left\{\left(T^{a}+1\right) \ln \left(k_{1} T^{l+m}\right)\right\}
$$


Next, we establish an upper bound for $E_{2}$.

Fix $\varepsilon$ and $\delta \in(0,1)$ until the completion of the argument. Denote $M_{\delta}:=\max _{s \in[0, \delta]} f(s)$. Given $u \in X_{T}$, set

$$
\widetilde{\nu}\left(u\left(t_{i-1}\right), u\left(t_{i}\right)\right)= \begin{cases}P_{l}\left(u\left(t_{i-1}\right)\right)^{l}, & \text { if } u\left(t_{i}\right)-u\left(t_{i-1}\right)=1, t_{i} \geq \delta T, \\ Q_{m}\left(u\left(t_{i-1}\right)\right)^{m}, & \text { if } u\left(t_{i}\right)-u\left(t_{i-1}\right)=-1, t_{i} \geq \delta T, \\ P_{l}\left(T\left(M_{\delta}+\varepsilon\right)\right)^{l}, & \text { if } u\left(t_{i}\right)-u\left(t_{i-1}\right)=1, t_{i}<\delta T, \\ Q_{m}\left(T\left(M_{\delta}+\varepsilon\right)\right)^{m}, & \text { if } u\left(t_{i}\right)-u\left(t_{i-1}\right)=-1, t_{i}<\delta T .\end{cases}
$$

As earlier, $t_{i}$ are the times of jumps in $u$.

If $\zeta_{T} \in U_{\varepsilon}(f)$ then, by (2) and the form of function $\widetilde{\nu}\left(u\left(t_{i-1}\right), u\left(t_{i}\right)\right)$, for $T$ sufficiently large and $t_{i-1}<\delta T$ we have an inequality

$$
\widetilde{\nu}\left(\zeta\left(t_{i-1}\right), \zeta\left(t_{i}\right)\right) \geq \nu\left(\zeta\left(t_{i-1}\right), \zeta\left(t_{i}\right)\right)
$$

Next, if $\zeta_{T} \in U_{\varepsilon}(f)$ and $\varepsilon$ is sufficiently small then for $s>\delta$ we have $\zeta_{T}(s)>\min _{s \in[\delta, 1]} f(s)-\varepsilon>0$. Thus, for $t_{i-1} \geq \delta T$ the condition (2) implies that for any $\gamma_{2} \in(0,1)$ and $T$ large enough

$$
\left(1-\gamma_{2}\right) \leq \frac{\nu\left(\zeta\left(t_{i-1}\right), \zeta\left(t_{i}\right)\right)}{\widetilde{\nu}\left(\zeta\left(t_{i-1}\right), \zeta\left(t_{i}\right)\right)} \leq\left(1+\gamma_{2}\right) .
$$

Owing to inequalities (29), (30) for any $\gamma_{2} \in(0,1)$ and $T$ sufficiently large we have that

$$
\begin{aligned}
& \prod_{i=1}^{N_{T}(\zeta)} \nu\left(\zeta\left(t_{i-1}\right), \zeta\left(t_{i}\right)\right) \mathbf{1}\left(\zeta_{T} \in U_{\varepsilon}(f), N_{T}(\zeta)>T^{a}\right) \\
& \quad \leq\left(1+\gamma_{2}\right)^{N_{T}(\zeta)} \prod_{i=1}^{N_{T}(\zeta)} \widetilde{\nu}\left(\zeta\left(t_{i-1}\right), \zeta\left(t_{i}\right)\right) \mathbf{1}\left(\zeta_{T} \in U_{\varepsilon}(f), N_{T}(\zeta)>T^{a}\right) .
\end{aligned}
$$

Next, set

$$
\widetilde{f}_{\delta}(s)= \begin{cases}M_{\delta}, & \text { if } t \in[0, \delta), \\ f(s), & \text { if } t \in[\delta, 1] .\end{cases}
$$

From the form of $\widetilde{\nu}\left(\zeta\left(t_{i-1}\right), \zeta\left(t_{i}\right)\right)$ it follows that for $\zeta_{T} \in U_{\varepsilon}(f)$ one of inequalities holds true, depending upon the sign of $\zeta\left(t_{i}\right)-\zeta\left(t_{i-1}\right)$ : either

$$
\widetilde{\nu}\left(\zeta\left(t_{i-1}\right), \zeta\left(t_{i}\right)\right) \leq P_{l}\left(T\left(\widetilde{f}_{\delta}\left(t_{i-1} / T\right)+\varepsilon\right)\right)^{l},
$$

or

$$
\widetilde{\nu}\left(\zeta\left(t_{i-1}\right), \zeta\left(t_{i}\right)\right) \leq Q_{m}\left(T\left(\widetilde{f}_{\delta}\left(t_{i-1} / T\right)+\varepsilon\right)\right)^{m} .
$$


If $\zeta_{T} \in U_{\varepsilon}(f)$ then, by virtue of (11), process $\zeta_{T}$ has $\frac{N_{T}(\zeta)+L}{2}$ positive jumps and $\frac{N_{T}(\zeta)-L}{2}$ negative jumps. Hence, from (31), (32) we obtain that

$$
\begin{aligned}
& \prod_{i=1}^{N_{T}(\zeta)} \nu\left(\zeta\left(t_{i-1}\right), \zeta\left(t_{i}\right)\right) \mathbf{1}\left(\zeta_{T} \in U_{\varepsilon}(f), N_{T}(\zeta)>T^{a}\right) \\
& \leq \begin{cases}\left(1+\gamma_{2}\right)^{N_{T}(\zeta)} T^{v L / 2} P_{l}^{\frac{N_{T}(\zeta)+L}{2}} Q_{m}^{\frac{N_{T}(\zeta)-L}{2}} \prod_{i=1}^{N_{T}(\zeta)} T^{\frac{l+m}{2}}(M+\varepsilon)^{v}, & \text { if } l \neq m, \\
\left(1+\gamma_{2}\right)^{N_{T}(\zeta)} P_{l}^{\frac{N_{T}(\zeta)+L}{2}} Q_{m}^{\frac{N_{T}(\zeta)-L}{2}} \prod_{i=1}^{N_{T}(\zeta)} T^{l}\left(\tilde{f}_{\delta}\left(t_{i-1} / T\right)+\varepsilon\right)^{l}, & \text { if } l=m .\end{cases}
\end{aligned}
$$

Set

$$
k_{2}(T):=\min \left(1,\left(P_{l} / Q_{m}\right)^{(f(1)-\varepsilon) T / 2}\right), k_{3}(T):=\max \left(1,\left(P_{l} / Q_{m}\right)^{(f(1)+\varepsilon) T / 2}\right) .
$$

Then from (10) it follows that

$$
k_{2}(T) \leq\left(\frac{P_{l}}{Q_{m}}\right)^{L / 2} \leq k_{3}(T)
$$
large

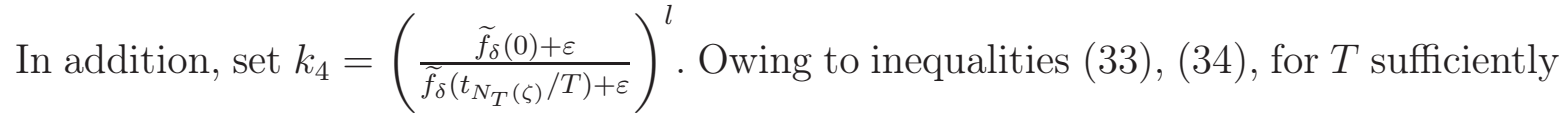

$$
E_{2} \leq \begin{cases}k_{3}(T) T^{\frac{v(M+\varepsilon)}{2}} \mathbf{E} \prod_{i=1}^{N_{T}(\zeta)} 2 P_{l}^{\frac{1}{2}} Q_{m}^{\frac{1}{2}}\left(1+\gamma_{2}\right) T^{(l+m) / 2}(M+\varepsilon)^{v}, & \text { if } l \neq m, \\ k_{3}(T) k_{4} \mathbf{E} \prod_{i=1}^{N_{T}(\zeta)} 2 P_{l}^{\frac{1}{2}} Q_{m}^{\frac{1}{2}}\left(1+\gamma_{2}\right) T^{l}\left(\tilde{f}_{\delta}\left(t_{i} / T\right)+\varepsilon\right)^{l}, & \text { if } l=m .\end{cases}
$$

Following Remark 4.2 from Appendix, we get an exponential bound for $E_{2}$ :

$$
E_{2} \leq \begin{cases}k_{3}(T) e^{-T} T^{\frac{v(M+\varepsilon)}{2}} \exp \left\{2 P_{l}^{\frac{1}{2}} Q_{m}^{\frac{1}{2}}\left(1+\gamma_{2}\right) T^{(l+m) / 2+1}(M+\varepsilon)^{v}\right\}, & \text { if } l \neq m, \\ k_{3}(T) k_{4} e^{-T} \exp \left\{2 P_{l}^{\frac{1}{2}} Q_{m}^{\frac{1}{2}}\left(1+\gamma_{2}\right) T^{l+1} \int_{0}^{1}\left(\tilde{f}_{\delta}(s)+\varepsilon\right)^{l} d s\right\}, & \text { if } l=m .\end{cases}
$$

Then, for $T$ sufficiently large, selecting $a<\frac{l+m}{2}+1$ we obtain from the bound (28) that

$$
\mathbf{E}\left(e^{B_{T}+N_{T}(\zeta) \ln 2} ; \zeta_{T} \in U_{\varepsilon}(f)\right)=E_{1}+E_{2} \leq 2 E_{2} .
$$

Finally, by taking into account that the value $\ln \left(k_{3}(T) T^{\frac{v(M+\varepsilon)}{2}}\right)$ is of order $T \ln T$, while $\ln \left(k_{3}(T) k_{4}\right)$ is of order $T$, we conclude: for any $\gamma_{2} \in(0,1)$ and $\delta \in(0,1)$, the following bounds hold true:

$$
\begin{aligned}
& \lim _{\varepsilon \rightarrow 0} \limsup _{T \rightarrow \infty} \frac{1}{T^{v+1}} \ln \mathbf{E}\left(e^{B_{T}+N_{T}(\zeta) \ln 2} ; \zeta_{T} \in U_{\varepsilon}(f)\right) \\
& \leq \begin{cases}0, & \text { if } l \neq m, \\
2 \sqrt{P_{l} Q_{m}}\left(1+\gamma_{2}\right) \int_{0}^{1} \widetilde{f}_{\delta}^{l}(s) d s, & \text { if } l=m .\end{cases}
\end{aligned}
$$


Taking the limit as $\gamma_{2}, \delta \rightarrow 0$ completes the proof.

Proof of Lemma 2.4. Let us now lower-bound the value $E_{2}$ from (27). As before, we fix a sufficiently small $\varepsilon$ until the end of the argument. Everywhere below, [ · stands for the integer part.

Introduce the event $D:=\left\{\max _{1 \leq k \leq N_{T}(\zeta)+1} \tau_{k} \leq T^{1-\beta}\right\}$, where $1<\beta<a$ and $\tau_{N_{T}(\zeta)+1}:=$ $T-t_{N_{T}(\zeta)}$. Also consider the event $C_{\varepsilon}:=\left\{\inf _{t \in\left[t_{[\varepsilon T / 4]}, T\right]} \zeta(t)>\varepsilon / 16\right\}$, where $t_{[\varepsilon T / 4]}$ is the time of the $[\varepsilon T / 4]$-th jump in $\zeta$.

Obviously

$$
\begin{aligned}
E_{2} & =2^{N_{T}(\zeta)} \mathbf{E} \prod_{i=1}^{N_{T}(\zeta)} \nu\left(\zeta\left(t_{i-1}\right), \zeta\left(t_{i}\right)\right) \mathbf{1}\left(\zeta_{T} \in U_{\varepsilon}(f), N_{T}(\zeta)>T^{a}\right) \\
& \geq \mathbf{E} 2^{N_{T}(\zeta)} \prod_{i=1}^{N_{T}(\zeta)} \nu\left(\zeta\left(t_{i-1}\right), \zeta\left(t_{i}\right)\right) \mathbf{1}\left(D, C_{\varepsilon}, \zeta_{T} \in U_{\varepsilon}^{+}(f), N_{T}(\zeta)>T^{a}\right),
\end{aligned}
$$

where $U_{\varepsilon}^{+}(f):=\left\{g: \min _{t \in[0,1]} g(t) \geq 0\right\} \cap U_{\varepsilon}(f)$.

Let $\delta=\min \left\{s: \min _{t \in[s, 1]} f(t) \geq 2 \varepsilon\right\}$ and denote $r(\delta):=\min \left\{i: t_{i} \geq T \delta\right\}$.

Suppose that $\zeta_{T} \in U_{\varepsilon}^{+}(f)$ and $r(\delta)+1 \leq i \leq N_{T}(\zeta)$. By condition (2), depending upon the sign of $\zeta\left(t_{i}\right)-\zeta\left(t_{i-1}\right)$, we have ether

$$
\nu\left(\zeta\left(t_{i-1}\right), \zeta\left(t_{i}\right)\right) \geq\left(1-\gamma_{3}\right) P_{l}\left(T\left(f\left(t_{i-1} / T\right)-\varepsilon\right)\right)^{l}
$$

or

$$
\nu\left(\zeta\left(t_{i-1}\right), \zeta\left(t_{i}\right)\right) \geq\left(1-\gamma_{3}\right) Q_{m}\left(T\left(f\left(t_{i-1} / T\right)-\varepsilon\right)\right)^{m} .
$$

If the event $C_{\varepsilon}$ has occurred, and $[\varepsilon T / 4] \leq i \leq r(\delta)$, then, owing to condition (2), for any $\gamma_{3} \in(0,1)$ and a sufficiently large $T$ the following inequality holds true:

$$
\nu\left(\zeta\left(t_{i-1}\right), \zeta\left(t_{i}\right)\right) \geq\left(1-\gamma_{3}\right)(T \varepsilon / 16)^{w}
$$

where $w:=\min (l, m)$.

For $\zeta_{T} \in U_{\varepsilon}^{+}(f)$ and $1 \leq i \leq[\varepsilon T / 4]$ we have

$$
\nu\left(\zeta\left(t_{i-1}\right), \zeta\left(t_{i}\right)\right) \geq k_{5}:=\min \left[\inf _{x \in \mathbb{Z}_{+}} \lambda(x), \inf _{x \in \mathbb{N}} \mu(x)\right] .
$$

Let us introduce the function

$$
\widehat{f}_{\varepsilon}(s)= \begin{cases}\frac{\varepsilon}{16 \max \left(1, P_{l}, Q_{m}\right)}, & \text { if } s \in[0, \delta), \\ f(s)-\varepsilon, & \text { if } s \in[\delta, 1] .\end{cases}
$$


Using (11), (35), (36), (37), (38), we get the bound

$$
\begin{aligned}
E_{2} \geq & k_{6}(T) \mathbf{E}\left[P_{l}^{\frac{N_{T}(\zeta)+L}{2}} Q_{m}^{\frac{N_{T}(\zeta)-L}{2}}\left(1-\gamma_{3}\right)^{N_{T}(\zeta)} 2^{N_{T}(\zeta)}\right. \\
& \left.\times \prod_{i=[\varepsilon T / 4]+1}^{N_{T}(\zeta)}\left(T \widehat{f}\left(t_{i-1} / T\right)\right)^{w} \mathbf{1}\left(D, C_{\varepsilon}, \zeta_{T} \in U_{\varepsilon}^{+}(f), N_{T}(\zeta)>T^{a}\right)\right],
\end{aligned}
$$

where $k_{6}(T):=\left(\frac{k_{5}}{\max \left(P_{l}, Q_{m}\right)}\right)^{[\varepsilon T / 4]}$.

From inequalities (10), (34) we obtain

$$
\begin{aligned}
E_{2} \geq & k_{7}(T) \mathbf{E}\left[P_{l}^{\frac{N_{T}(\zeta)}{2}} Q_{m}^{\frac{N_{T}(\zeta)}{2}}\left(1-\gamma_{3}\right)^{N_{T}(\zeta)} 2^{N_{T}(\zeta)}\right. \\
& \left.\times \prod_{i=1}^{N_{T}(\zeta)}\left(T \widehat{f}\left(t_{i} / T\right)\right)^{w} \mathbf{1}\left(D, C_{\varepsilon}, \zeta_{T} \in U_{\varepsilon}^{+}(f), N_{T}(\zeta)>T^{a}\right)\right],
\end{aligned}
$$

where $k_{7}(T):=\frac{k_{6}(T) k_{2}(T)}{M^{w} T^{w[\varepsilon T / 4]}}$.

From Lemma 4.4 of Appendix it follows that for any $\gamma_{4} \in(0,1)$ and $T$ sufficiently large the following holds true

$$
E_{2} \geq k_{7}(T) \sum_{n=\left[T^{a}\right]+1}^{\infty} 2^{n}\left(1-\gamma_{4}\right)^{n} P_{l}^{\frac{n}{2}} Q_{m}^{\frac{n}{2}} \mathbf{E} \prod_{i=1}^{n} T^{w}\left(\widehat{f}\left(t_{i} / T\right)\right)^{w} \mathbf{1}\left(D, N_{T}(\zeta)=n\right)
$$

Here $\gamma_{4}$ is expressed via $\gamma_{3}$ and $\theta$ whereas $\theta \in(0,1)$ is introduced in Lemmas 4.3, 4.4 from Appendix.

To estimate the product from (39), we use Lemma 4.1. Taking into account that $n>T^{a}$, we get that for $T$ large enough,

$$
\begin{gathered}
\mathbf{E}\left[\prod_{i=1}^{n} T^{w}\left(\widehat{f}\left(t_{i} / T\right)\right)^{w} \mathbf{1}\left(D, N_{T}(\zeta)=n\right)\right]=\mathbf{E}\left[\prod_{i=1}^{n} T^{w}\left(\widehat{f}\left(t_{i} / T\right)\right)^{w} \mathbf{1}\left(N_{T}(\zeta)=n\right)\right] \\
-\mathbf{E}\left[\prod_{i=1}^{n} T^{w}\left(\widehat{f}\left(t_{i} / T\right)\right)^{w} \mathbf{1}\left(\bar{D}, N_{T}(\zeta)=n\right)\right] \\
\quad \geq \frac{\left(T^{w} \int_{0}^{T}\left(\widehat{f}\left(t_{i} / T\right)\right)^{w} d t\right)^{n}}{n !} e^{-T}-2 T^{\beta} \frac{\left(T^{w} \int_{0}^{T}\left(\widehat{f}\left(t_{i} / T\right)\right)^{w} d t-T^{w+1} \alpha_{1 / T^{\beta}}\right)^{n}}{n !} e^{-T} .
\end{gathered}
$$

Here $\alpha_{1 / T^{\beta}}=\frac{1}{2 T^{\beta}} \inf _{s \in[0,1]}(\widehat{f}(s))^{w}=\frac{1}{2 T^{\beta}}\left(\frac{\varepsilon}{16 \max \left(1, P_{l}, Q_{m}\right)}\right)^{w}$ (compare to equation (43) in Appendix) .

Let us now estimate the last summand in the right side of (401). Denote $k_{8}:=\sup _{s \in[0,1]}(\widehat{f}(s))^{w}$. 
As $a>\beta$, for a sufficiently large $T$ the following inequalities hold true:

$$
\begin{aligned}
2 T^{\beta} & \left(T^{w} \int_{0}^{T}(\widehat{f}(t / T))^{w} d t-T^{w+1} \alpha_{1 / T^{\beta}}\right)^{n} \\
& \left.\leq 2 T^{\beta}\left(T^{w} \int_{0}^{T}(\widehat{f}(t / T))^{w} d t\right)^{n}\left(1-\frac{\varepsilon^{w}}{2 k_{8} T^{\beta}\left(16 \max \left(1, P_{l}, Q_{m}\right)\right)^{w}}\right)^{n}\right)^{T^{a}} \\
& \leq 2 T^{\beta}\left(T^{w} \int_{0}^{T}(\widehat{f}(t / T))^{w} d t\right)^{n}\left(1-\frac{\varepsilon^{w}}{2 k_{8} T^{\beta}\left(16 \max \left(1, P_{l}, Q_{m}\right)\right)^{w}} \varepsilon^{w} T^{a-\beta}\right) \\
& \leq\left(T^{w} \int_{0}^{T}(\widehat{f}(t / T))^{w} d t\right)^{n} \exp \left(\beta \ln (2 T)-\frac{\varepsilon^{w}\left(16 \max \left(1, P_{l}, Q_{m}\right)\right)^{w}}{2 k_{8}(16)}\right. \\
& \leq \frac{1}{2}\left(T^{w} \int_{0}^{T} \widehat{f}^{w}(t / T) d t\right)^{n} .
\end{aligned}
$$

Consequently, from (40) it follows that

$$
\mathbf{E}\left[\prod_{i=1}^{n} T^{w}\left(\widehat{f}\left(t_{i} / T\right)\right)^{w} \mathbf{1}\left(D, N_{T}(\zeta)=n\right)\right] \geq \frac{1}{2} \frac{\left(T^{w} \int_{0}^{T}(\widehat{f}(t / T))^{w} d t\right)^{n}}{n !} e^{-T}
$$

By virtue of (39), for $T$ sufficiently large,

$$
E_{2} \geq \frac{k_{7}(T)}{2} \sum_{n=\left[T^{a}\right]+1}^{\infty} 2^{n}\left(1-\gamma_{4}\right)^{n} \sqrt{P_{l} Q_{m}} \frac{\left(T^{w} \int_{0}^{T}(\widehat{f}(t / T))^{w} d t\right)^{n}}{n !} e^{-T}
$$

From this it follows that, selecting $a<w+1$, for $T$ large enough we obtain the inequalities

$$
\begin{gathered}
E_{2} \geq \frac{k_{7}(T) e^{-T}}{2} \exp \left(2\left(1-\gamma_{4}\right) \sqrt{P_{l} Q_{m}} T^{w+1} \int_{0}^{1} \widehat{f}(s)^{w} d s\right) \\
-\frac{k_{7}(T) e^{-T}}{2} \exp \left(a \ln (T)+(w+2) T^{a} \ln (T)\right) \\
\geq \frac{k_{7}(T) e^{-T}}{4} \exp \left(2\left(1-\gamma_{4}\right) \sqrt{P_{l} Q_{m}} T^{w+1} \int_{0}^{1} \widehat{f}(s)^{w} d s\right) .
\end{gathered}
$$

By virtue of (41) and the fact that $\ln k_{7}$ is a quantity of order $T \ln T$, we now obtain that

$$
\begin{aligned}
\liminf _{T \rightarrow \infty} \frac{1}{T^{v+1}} \ln \mathbf{E}\left(e^{B_{T}+N_{T}(\zeta) \ln 2} ; \zeta_{T} \in U_{\varepsilon}(f)\right) \\
\quad \geq \begin{cases}0, & \text { if } l \neq m, \\
2\left(1-\gamma_{4}\right) \sqrt{P_{l} Q_{m}} \int_{0}^{1} \widehat{f}(s)^{l} d s, & \text { if } l=m .\end{cases}
\end{aligned}
$$

Furthermore, taking into account the definition of function $\widehat{f}(s)$, we obtain that

$$
\begin{aligned}
\lim _{\varepsilon \rightarrow 0} \liminf _{T \rightarrow \infty} \frac{1}{T^{v+1}} \ln \mathbf{E}\left(e^{B_{T}+N_{T}(\zeta) \ln 2} ; \zeta_{T} \in U_{\varepsilon}(f)\right) & \\
& \geq \begin{cases}0, & \text { if } l \neq m, \\
2\left(1-\gamma_{4}\right) \sqrt{P_{l} Q_{m}} \int_{0}^{1} f^{l}(s) d s, & \text { if } l=m .\end{cases}
\end{aligned}
$$

Taking the limit as $\delta \rightarrow 0$ and $\gamma_{4} \rightarrow 0$ completes the proof of the lemma. 


\section{Appendix}

In this section we prove the auxiliary assertions used in earlier arguments.

Let $X_{T}^{(n)}$ stand for the event that process $\zeta$ has exactly $n$ jumps on the interval $[0, T]$.

Lemma 4.1 Let $g(t)$ be a non-negative bounded Borel function and $n \geq 1$. Then

$$
\mathbf{E}\left[\prod_{i=1}^{n} g\left(t_{i}\right) \mathbf{1}\left(X_{T}^{(n)}\right)\right]=\frac{\left(\int_{0}^{T} g(s) d s\right)^{n}}{n !} e^{-T}
$$

and

$$
\mathbf{E}\left[\prod_{i=1}^{n} g\left(t_{i}\right) \mathbf{1}\left(X_{T}^{(n)}\right) \mathbf{1}\left(\max _{1 \leq k \leq n+1} \tau_{k}>T \Delta\right)\right] \leq \frac{2}{\Delta} \frac{\left(\int_{0}^{T} g(s) d s-T \alpha_{\Delta}\right)^{n}}{n !} e^{-T}
$$

Here $\Delta>0$ is a constant and $\alpha_{\Delta}:=\frac{\Delta}{2} \inf _{t \in[0, T]} g(t)$. Further, $t_{1}, \ldots, t_{n}$ are jump times on $[0, T]$ in process $\zeta$ and $\tau_{n+1}:=T-t_{n}$.

Proof. First, we prove (42). To this end, write:

$$
\mathbf{E}\left(\prod_{i=1}^{n} g\left(t_{i}\right) \mid X_{T}^{(n)}\right)=\mathbf{E}\left(\prod_{i=1}^{n} g\left(t_{i}\right) \mid \eta(T)=n\right)
$$

where $\eta$ is a Poisson process with mean $\mathbf{E} \eta(t)=t$.

From [14], Theorem 2.3, p. 126, it follows that

$$
\begin{aligned}
& \mathbf{E}\left(\prod_{i=1}^{n} g\left(t_{i}\right) \mid \eta(T)=n\right) \\
& \quad=\frac{n !}{T^{n}} \int_{0}^{T}\left(\int_{s_{1}}^{T} \ldots\left(\int_{s_{n-1}}^{T} \prod_{i=1}^{n} g\left(s_{i}\right) d s_{n}\right) \ldots d s_{2}\right) d s_{1}=\frac{1}{T^{n}}\left(\int_{0}^{T} g(s) d s\right)^{n} .
\end{aligned}
$$

Therefore,

$$
\mathbf{E}\left[\prod_{i=1}^{n} g\left(t_{i}\right) \mathbf{1}\left(X_{T}^{(n)}\right)\right]=\frac{1}{T^{n}}\left(\int_{0}^{T} g(s) d s\right)^{n} \mathbf{P}(\eta(T)=n)=\frac{1}{n !}\left(\int_{0}^{T} g(s) d s\right)^{n} e^{-T} .
$$

Next, we turn to the proof of (43). Here

$$
\begin{aligned}
\mathbf{E} & {\left[\prod_{i=1}^{n} g\left(t_{i}\right) \mathbf{1}\left(X_{T}^{(n)}\right) \mathbf{1}\left(\max _{1 \leq k \leq n+1} \tau_{k}>T \Delta\right)\right] } \\
& \leq \sum_{r=1}^{[2 / \Delta]} \mathbf{E}\left[\prod_{i=1}^{n} g\left(t_{i}\right) \mathbf{1}(\eta(T)=n) \mathbf{1}\left(\eta\left(\frac{r T \Delta}{2}\right)-\eta\left(\frac{(r-1) T \Delta}{2}\right)=0\right)\right]:=\sum_{r=1}^{[2 / \Delta]} D_{r} .
\end{aligned}
$$

Using the fact that $\max _{1 \leq k \leq n+1} \tau_{k}>T \Delta$, we get that there exists an $r$ with $1 \leq r \leq\left[\frac{2}{\Delta}\right]$ and with no jumps on interval $\left[\frac{(r-1) T \Delta}{2}, \frac{r T \Delta}{2}\right]$. 
Write

$$
\begin{aligned}
D_{1} & =\mathbf{E}\left[\prod_{i=1}^{n} g\left(t_{i}\right) \mathbf{1}(\eta(T)=n) \mathbf{1}\left(\eta\left(\frac{T \Delta}{2}\right)=0\right)\right] \\
& =\mathbf{E}\left[\prod_{i=1}^{n} g\left(t_{i}\right) \mathbf{1}\left(\eta(T)-\eta\left(\frac{T \Delta}{2}\right)=n\right) \mathbf{1}\left(\eta\left(\frac{T \Delta}{2}\right)=0\right)\right] .
\end{aligned}
$$

By using the independence of increments in, and the homogeneity of, the Poisson process and formula (42) we obtain

$$
D_{1}=\frac{\left(\int_{\frac{T \Delta}{2}}^{T} g(s) d s\right)^{n}}{n !} e^{-T(1-\Delta / 2)} \mathbf{P}\left(\eta\left(\frac{T \Delta}{2}\right)=0\right)=\frac{\left(\int_{\frac{T \Delta}{2}}^{T} g(s) d s\right)^{n}}{n !} e^{-T} .
$$

Similarly for any $1 \leq r \leq\left[\frac{2}{\Delta}\right]$ one obtains that

$$
D_{r}=\frac{\left(\int_{[0, T] \backslash B_{r, \Delta}} g(s) d s\right)^{n}}{n !} e^{-T},
$$

where $B_{r, \Delta}=\left[\frac{(r-1) T \Delta}{2}, \frac{r T \Delta}{2}\right]$.

In view of the relations

$$
\min _{1 \leq r \leq\left[\frac{2}{\Delta}\right]} \int_{\left[\frac{(r-1) T \Delta}{2}, \frac{r T \Delta}{2}\right]} g(s) d s \geq T \frac{\Delta}{2} \inf _{s \in[0, T]} g(s)=T \alpha_{\Delta},
$$

we get that

$$
\mathbf{E}\left[\prod_{i=1}^{n} g\left(t_{i}\right) \mathbf{1}\left(X_{T}^{(n)}\right) \mathbf{1}\left(\max _{1 \leq k \leq n+1} \tau_{k}>T \Delta\right)\right] \leq \frac{2}{\Delta} \frac{\left(\int_{0}^{T} g(s) d s-T \alpha_{\Delta}\right)^{n}}{n !} e^{-T} .
$$

Remark 4.2 Lemma 4.1 implies that

$$
\mathbf{E} \prod_{i=1}^{\eta(T)} g\left(t_{i}\right) \mathbf{1}(\eta(T) \geq 1)=e^{-T}\left(\exp \left\{\int_{0}^{T} g(s) d s\right\}-1\right) .
$$

Lemma 4.3 Consider a sequence $b_{1}, b_{2}, \ldots, b_{n}$, where each $b_{i}$ equals -1 or 1 . Define by $c_{d}$ the number of sequences with following property:

$$
\left|\sum_{k=1}^{r} b_{k}\right| \leq d, \forall 1 \leq r \leq n .
$$

Take $d=[T \Delta]$ and $n=O\left(T^{\beta}\right)$ where $T \rightarrow \infty$ while $\Delta>0, \beta>1$ are fixed. Then for any $\theta \in(0,1)$ and sufficiently large $T$ we hjave the bound

$$
c_{d} \geq(1-\theta)^{n+1} 2^{n} .
$$


ProOF. It is clear that if a sequence $b_{2(p-1) d+1}, \ldots, b_{2 p d}$, with $1 \leq p \leq \frac{n}{2 d}$, has an equal number of 1 and -1 , and in the sequence $b_{2 d\left[\frac{n}{2 d}\right]+1}, \ldots, b_{n}$ the difference between the numbers of 1 and -1 in the absolute value is at most 1 then required property is fulfilled. The number of such sequences is not less then $\left(C_{2 d}^{d}\right)^{\left[\frac{n}{2 d}\right]}$.

Using Stirling's formula gives that

$$
\left(C_{2 d}^{d}\right)^{\left[\frac{n}{2 d}\right]} \sim\left(\frac{\sqrt{2}(2 d)^{2 d}}{\sqrt{\pi d} d^{2 d}}\right)^{\left[\frac{n}{2 d}\right]}=\left(\frac{\sqrt{2} 2^{2 d}}{\sqrt{\pi d}}\right)^{\left[\frac{n}{2 d}\right]} \geq 2^{n-2 d}(\pi d)^{-\frac{n}{4 d}}
$$

Thus, owing to the fact that $-2 d \ln 2-\frac{n r d}{4 d}=o(n)$, we obtain that, for any $\theta \in(0,1)$ and $T$ sufficiently large,

$$
c_{d} \geq(1-\theta) 2^{n} \exp \left(-2 d \ln 2-\frac{n \ln \pi d}{4 d}\right) \geq(1-\theta)^{n+1} 2^{n} .
$$

Lemma 4.4 Take $\beta>1$ and $n \geq T^{\beta}$ and let $g(\cdot)$ be a non-negative bounded Borel function. For any $\theta>0$ and all $T$ sufficiently large the following estimate holds true

$$
\begin{aligned}
& \mathbf{E}\left[g\left(t_{1}, \ldots, t_{n}\right) \mathbf{1}\right.\left.\left(\max _{1 \leq k \leq n+1} \tau_{k} \leq T^{1-\beta}\right) \mathbf{1}\left(X_{T}^{(n)}\right) \mathbf{1}\left(\zeta_{T} \in U_{\varepsilon}^{+}(f)\right) \mathbf{1}\left(C_{\varepsilon}\right)\right] \\
& \geq(1-\theta)^{2 n} \mathbf{E}\left[g\left(t_{1}, \ldots, t_{n}\right) \mathbf{1}\left(\max _{1 \leq k \leq n+1} \tau_{k} \leq T^{1-\beta}\right) \mathbf{1}\left(X_{T}^{(n)}\right)\right]
\end{aligned}
$$

Here $U_{\varepsilon}^{+}(f):=\left\{g: \min _{t \in[0,1]} g(t) \geq 0\right\} \cap U_{\varepsilon}(f), C_{\varepsilon}:=\left\{\inf _{t \in\left[t_{[\varepsilon T / 4]}, T\right]} \zeta(t)>\varepsilon / 16\right\}$ and $t_{[\varepsilon T / 4]}$ is the point of the $[\varepsilon T / 4]-$ th jump in process $\zeta$.

Proof As $f$ is uniformly continuous on $[0,1]$, then for $\delta>0$ sufficiently small we have the inequality

$$
\sup _{s, t:|s-t| \leq \delta}|f(s)-f(t)|<\frac{\varepsilon}{4} .
$$

Fix $\delta$ with $1 / \delta \in \mathbb{N}$ and let $1 \leq r \leq 1 / \delta$.

Denote by $B_{m_{r}, \delta r}$ the event where process $\zeta$ has exactly $m_{r}$ jumps on the interval $[T \delta(r-1), T \delta r]$.

Then we can write

$$
\begin{aligned}
& \mathbf{E}\left[g\left(t_{1}, \ldots, t_{n}\right) \mathbf{1}\left(\max _{1 \leq k \leq n+1} \tau_{k} \leq T^{1-\beta}\right) \mathbf{1}\left(X_{T}^{(n)}\right) \mathbf{1}\left(\zeta_{T} \in U_{\varepsilon}^{+}(f)\right) \mathbf{1}\left(C_{\varepsilon}\right)\right] \\
& =\sum_{m_{1}, \ldots, m_{1 / \delta}} \mathbf{E}\left[g\left(t_{1}, \ldots, t_{n}\right) \mathbf{1}\left(\max _{1 \leq k \leq n+1} \tau_{k} \leq T^{1-\beta}\right) \mathbf{1}\left(X_{T}^{(n)}\right) \mathbf{1}\left(\zeta_{T} \in U_{\varepsilon}^{+}(f)\right) \mathbf{1}\left(C_{\varepsilon}\right) \prod_{r=1}^{1 / \delta} \mathbf{1}\left(B_{m_{r}, \delta r}\right)\right] .
\end{aligned}
$$

The summation here is over all collections with $\min _{r} m_{r} \geq \delta T^{\beta}, \sum_{r} m_{r}=n$. 
Take a collection $m_{1}, \ldots, m_{r}$ satisfying the above condition.

Consider a piece of a trajectory of $\zeta$ on the interval $[0, \delta T]$. Denote by $t_{1,1}, \ldots, t_{m_{1}, 1}$ the jump points of $\zeta$ lying in this interval. Suppose that the jumps satisfy the following conditions:

1) The jumps at times $t_{1,1}, \ldots, t_{d_{\varepsilon, 1}, 1}$ are positive where $d_{\varepsilon, 1}:=[T \varepsilon / 4]$.

2) The jumps at times $t_{d_{\varepsilon, 1}+1,1}, \ldots, t_{m_{1}, 1}$ are such that for any integer $k \in\left[d_{\varepsilon, 1}+1, m_{1}\right]$ we have the inequality

$$
\left|\sum_{l=d_{\varepsilon, 1}+1}^{k} \zeta\left(t_{l, 1}\right)\right| \leq\left[\frac{T \varepsilon}{8}\right] .
$$

Then, for $T$ large enough, the trajectory $\zeta_{T}(t)$ has the following properties.

1) The trajectory is non-negative and lies in an $\varepsilon$-neighborhood of function $f$ for $t \in[0, \delta]$.

2) $\zeta_{T}(t) \geq \varepsilon / 16$ for $t>t_{d_{\varepsilon, 1}, 1} / T$.

3) $\left|\zeta_{T}(\delta)-f(\delta)\right| \leq 3 \varepsilon / 8$.

Now consider a piece of a trajectory of $\zeta$ defined on the interval $[\delta T, 2 \delta T]$ and having the property $|\zeta(\delta T)-T f(\delta)| \leq 3 \varepsilon T / 8$. Denote by $t_{1,2}, \ldots, t_{m_{2}, 2}$ the jump points of $\zeta$ in this interval.

Let these jumps satisfy the conditions:

1) At times $t_{1,2}, \ldots, t_{\left|d_{\varepsilon, 2}\right|, 2}$ the jumps are positive or negative in accordance with the sign of the value $d_{\varepsilon, 2}:=\left[T\left(\max (\varepsilon / 4, f(2 \delta))-\zeta_{T}(\delta)\right)\right]$.

2) At times $t_{\left|d_{\varepsilon, 2}\right|+1,2}, \ldots, t_{m_{2}, 2}$ are such that for any integer $k \in\left[\left|d_{\varepsilon, 2}\right|+1, m_{2}\right]$ the following inequality holds true:

$$
\left|\sum_{l=\left|d_{\varepsilon, 2}\right|+1}^{k} \zeta\left(t_{l, 2}\right)\right| \leq\left[\frac{T \varepsilon}{8}\right] .
$$

Then, again for $T$ large enough, the trajectory $\zeta_{T}(t)$ has the following properties.

1) The trajectory is non-negative and lies in a $\varepsilon$-neighborhood of $f$ for $t \in[\delta, 2 \delta]$.

2) $\zeta_{T}(t) \geq \varepsilon / 16$ as $t \in[\delta, 2 \delta]$.

3) $\left|\zeta_{T}(2 \delta)-f(2 \delta)\right| \leq 3 \varepsilon / 8$.

Further pieces of the trajectory are dealt with by induction.

Let us count the trajectories whose jumps satisfy the above properties.

As $\max _{1 \leq k \leq n+1} \tau_{k} \leq T^{1-\beta}$, we have that for any $r$ with $1 \leq r \leq 1 / \delta$ the interval $[T \delta(r-$ 1), $T \delta r]$ contains at least $\left[\delta T^{\beta}\right]$ jumps of process $\zeta$, where $\beta>1$. Using Lemma 4.3 yields that, when $T$ is sufficiently large, we will have on $[T \delta(r-1), T \delta r]$ a number of pieces of the trajectory with the aforementioned properties which is not less than

$$
(1-\theta)^{m_{r}+1-\left|d_{\varepsilon, r}\right|} 2^{m_{r}-\left|d_{\varepsilon, r}\right|}>(1-\theta)^{2 m_{r}} 2^{m_{r}} .
$$


Consequently, the number of trajectories that fulfill the above properties for all $r$ is not less then

$$
\prod_{r}(1-\theta)^{2 m_{r}} 2^{m_{r}}=(1-\theta)^{2 n} 2^{n}
$$

Next, the jump directions in $\zeta$ are mutually independent and do not depend either on the number of jumps within the interval or on the jump times. Hence, we can use equality (44) and get that

$$
\begin{aligned}
& \sum_{m_{1}, \ldots, m_{1 / \delta}} \mathbf{E}\left[g\left(t_{1}, \ldots, t_{n}\right) \mathbf{1}\left(\max _{1 \leq k \leq n+1} \tau_{k} \leq T^{1-\beta}\right) \mathbf{1}\left(X_{T}^{(n)}\right) \mathbf{1}\left(\zeta_{T} \in U_{\varepsilon}^{+}(f)\right) \mathbf{1}\left(C_{\varepsilon}\right) \prod_{r=1}^{1 / \delta} \mathbf{1}\left(B_{m_{r}, \delta r}\right)\right] \\
& \quad \geq \sum_{m_{1}, \ldots, m_{1 / \delta}} \frac{(1-\theta)^{2 n} 2^{n}}{2^{n}} \mathbf{E}\left[g\left(t_{1}, \ldots, t_{n}\right) \mathbf{1}\left(\max _{1 \leq k \leq n+1} \tau_{k} \leq T^{1-\beta}\right) \prod_{r=1}^{1 / \delta} \mathbf{1}\left(B_{m_{r}, \delta r}\right)\right] \\
& \quad=(1-\theta)^{2 n} \mathbf{E}\left[g\left(t_{1}, \ldots, t_{n}\right) \mathbf{1}\left(\max _{1 \leq k \leq n+1} \tau_{k} \leq T^{1-\beta}\right) \mathbf{1}\left(X_{T}^{(n)}\right)\right] .
\end{aligned}
$$

\section{Acknowledgments}

The authors are grateful to A.A. Borovkov, B.M. Gurevich, A.M. Mogulsky and E.A. Pechersky for their interest and useful comments. E.A. Pechersky should be credited for an initial statement of the probem. We also thank the anonymous referees for critical remarks and suggestions.

AVL thanks FAPESP for the financial support via Grant 2017/20482. YMS thanks Math Department, Penn State University, for hospitality and support and StJohn's College, Cambridge, for support. AAY thanks CNPq and FAPESP for the financial support via Grants 301050/2016-3 and 2017/10555-0, respectively.

\section{Список литературы}

[1] A. Dembo, O. Zeitouni. Large Deviations Techniques and Applications. New York, Springer. 1998.

[2] J.D. Deuschel, D.W. Stroock. Large Deviations. AMS Chelsea Publishing, Providence, RI, 1989.

[3] F. den Hollander. Large Deviations. Fields Institute Monographs, 14. American Mathematical Society, Providence, RI, 2000. 
[4] E. Olivieri, M.E. Vares. Large Deviations and Metastability. Cambridge University Press, Cambridge, 2005.

[5] A. Puhalskii. Large Deviations and Idempotent Probability. Chapman \& Hall/CRC. Boca Raton, 2001.

[6] S.R. Varadhan. Large Deviations and Applications. New York, SIAM,1984.

[7] Y. Suhov, I. Stuhl. On principles of large deviation and selected data compression. arXiv:1604.06971v1. 2015; Also: I. Stuhl, Y. Suhov, Selected data compression: a refinement of Shannon's principle In: Analytical and Computational Methods in Probability Theory. Lecture Notes in Computer Science, 10684. Springer, Berlin, 2017, PP. 309-321.

[8] M. Kelbert, I. Stuhl, Y. Suhov. Weighted entropy and optimal portfolios for risk-averse Kelly investments. Aequationes Mathematicae, 92 No 1 (2018), 165-200.

[9] A. Mazel, Y. Suhov, I. Stuhl, S. Zohren. Dominance of most tolerant species in multitype lattice Widom-Rowlinson models. Journ. Stat. Mech., 2014. 8-10.

[10] A. Mogulsky, E. Pechersky, A. Yambartsev. Large deviations for excursions of nonhomogeneous Markov processes. Electronic Commun. Probab., 19 (2014), 1-8.

[11] N. Vvedenskaya, Y. Suhov, V. Belitsky. A non-linear model of trading mechanism on a financial market. Markov Processes. Rel. Fields, 19 No 1 (2013), 83-98. arXiv:1201.4580. 2012 .

[12] W. Feller. An Introduction to Probability Theory and Its Applications, Vol. 2. Wiley, New York, 1971.

[13] M.Kelbert, Y. Suhov. Probability and Statistics by Example, Vol. 2. Cambridge University Press, Cambridge, 2008.

[14] S. Karlin, H. M. Taylor. A First Course in Stochastic Processes, 2nd Edition. New York et al., Academic Press, 1975.

[15] V.S. Korolyuk, N.I. Portenko, A.V. Skorokhod, A.F. Turbin. Handbook of Probability Theory and Mathematical Statistics. Nauka, Moscow, 1985.

[16] Dynkin E.B. Markov Processes, vols 1, 2. Academic Press, New York and Springer, Berlin, 1965. 
[17] K. Ito. Stochastic Processes, Vol. 2. Nauka, Moscow, 1963 (Russian). Also see K. Ito. Essentials of Stochastic Processes. Americal Mathematical Society, Providence, RI, 2006, and K. Ito. Stochastic Processes. Springer, Berlin et al., 2004.

[18] S. Karlin, J. McGregor. The classification of birth and death processes. Trans. Amer. Math. Soc., 86 (1957), 366-400.

[19] W. Ledermann, G.E.H. Reuter. Spectral theory for the differential equations of simple birth and death processes. Philos. Trans. Roy. Soc. London, Ser. A, 246, (1954), 321369.

[20] J.R. Norris. Markov Chains. Cambridge. Cambridge University Press, 1997.

[21] D. Stroock. An Introduction to Markov Processes, 2nd Ed. Springer, Heidelberg et al., 2014.

[22] A.A. Borovkov, A.A. Mogulskii. On large deviation principles in metric spaces. Siberian Math. Journ., 51 No 6 (2010), 989-1003.

[23] A.A. Borovkov, A.A. Mogulskii. Large deviation principles for random walk trajectories I. Theory Probab. Appl., 56 No 4 (2011), 538-561.

[24] A.V. Logachov. The local principle of large deviations for solutions of Itô stochastic equations with quick drift. Journ. Math. Sci., 218 No 1 (2016), 28-38.

[25] A.A. Borovkov, A.A. Mogulski. Inequalities and principle of large deviations for the trajectories of processes with independent increments. Siberian Math. Journ., $\mathbf{5 4}$ No 2 (2013), 217-226.

[26] N.Dunford, J.Schwartz. Linear operators. General theory. Wiley-Blackwell, Hoboken, NJ, 1988.

[27] W. Rudin. Real and Complex Analysis. McGrow-Hill, 1987. 